\section{Screening can identify unsuspected coronary stenoses after mediastinal irradiation}

Incidental cardiac irradiation during treatment for mediastinal neoplasms has been associated with an increased risk of subsequent death from ischemic and other heart diseases. Survival times are increasing following the treatment of thoracic malignancies, and consequently the population at risk of radiation-induced coronary artery disease (CAD), acute myocardial infarction or sudden cardiac death is growing. Researchers from the Stanford University Medical Center, CA, report that stress imaging can identify severe coronary stenoses in asymptomatic individuals who have previously received mediastinal irradiation.

Heidenreich et al. enrolled 294 outpatients who had received mediastinal irradiation doses of $\geq 35$ Gy for Hodgkin's disease. At enrollment, none of the patients had known ischemic cardiac disease. All participants underwent stress echocardiography and 274 patients underwent radionuclide perfusion imaging at the same stress session. Abnormal ventricular images at rest-indicative of prior myocardial injurywere found in 63 of the participants. During stress testing 54 participants exhibited perfusion defects, impaired wall motion, or both. Following these imaging results, coronary angiography was performed in 40 patients. This procedure revealed stenosis of $\geq 50 \%$ in 22 patients, with vessel narrowing reaching $\geq 70 \%$ in 16 of these individuals. Over a median followup of 6.5 years, symptomatic CAD was documented in 23 study participants, 10 of whom had an acute myocardial infarction.

These results indicate a high prevalence of unsuspected coronary stenoses in patients who have undergone mediastinal irradiation. The authors suggest that clinicians should consider screening for CAD in patients who have received mediastinal irradiation at doses of $35 \mathrm{~Gy}$ or more.

Original article Heidenreich PA et al. (2007) Screening for coronary artery disease after mediastinal irradiation for Hodgkin's disease. J Clin Oncol 25: 43-49

\section{Guidelines: carotid artery disease screening}

The Multidisciplinary Practice Guidelines Committee of the American Society of
Neuroimaging and the Society of Vascular and Interventional Neurology have released guidelines outlining which patient and population groups should be screened for asymptomatic extracranial carotid artery stenosis. The committee categorized the value of screening on the basis of the expected prevalence of carotid artery stenosis within the group, the anticipated benefit from intervention according to ACAS, ACST and SAPPHIRE trial results, and existing recommendations.

Within these comprehensive guidelines the authors strongly recommend screening of all patients with symptomatic peripheral vascular disease, and of patients with specified risk factors who are due to undergo CABG surgery. They also recommend screening of patients with abdominal aortic aneurysm with a history of transient ischemic attack, ischemic stroke, or retinal ischemic events. Patients who develop any of the last three conditions following carotid endarterectomy or carotid stent placement or have known contralateral untreated carotid stenosis should also be screened, but otherwise routine screening following these procedures is not recommended. All patients who have received unilateral or bilateral irradiation to the neck for head or neck cancer should be screened 10 years after treatment. Within the general population, the authors advocate possible screening of selected individuals aged 65 years or more who meet at least three of the following criteria: hypertension, coronary artery disease, hyperlipidemia, or current cigarette smoking.

Original article Qureshi AI et al. (2007) Guidelines for screening of extracranial carotid artery disease: a statement for healthcare professionals from the Multidisciplinary Practice Guidelines Committee of the American Society of Neuroimaging; cosponsored by the Society of Vascular and Interventional Neurology. J Neuroimaging 17: 19-47

\section{A simple score can predict 2-day stroke risk after transient ischemic attack}

Patients presenting with transient ischemic attack (TIA) have a high risk of subsequent stroke, especially in the very short term. In this study, Johnston and co-workers have evaluated two existing scores, the California and $A B C D$ scores, which are optimized to predict 90-day and 7-day risks of stroke after TIA, 